\title{
Low-temperature behavior of two-dimensional Gaussian Ising spin glasses
}

\author{
Jérôme Houdayer \\ Service de Physique Théorique, CEA Saclay, 91191 Gif-sur-Yvette, France \\ Alexander K. Hartmann
Institut für Theoretische Physik, Universität Göttingen, Tammannstraße 1, 3707ry Göttingen, Germany
}

(Dated: November 12, 2018)

\begin{abstract}
We perform Monte Carlo simulations of large two-dimensional Gaussian Ising spin glasses down to very low temperatures $\beta=1 / T=50$. Equilibration is ensured by using a cluster algorithm including Monte Carlo moves consisting of flipping fundamental excitations. We study the thermodynamic behavior using the Binder cumulant, the spin-glass susceptibility, the distribution of overlaps, the overlap with the ground state and the specific heat. We confirm that $T_{c}=0$. All results are compatible with an algebraic divergence of the correlation length with an exponent $\nu$. We find $-1 / \nu=-0.295(30)$, which is compatible with the value for the domain-wall and droplet exponent $\theta \approx-0.29$ found previously in ground-state studies. Hence the thermodynamic behavior of this model seems to be governed by one single exponent.
\end{abstract}

PACS numbers: 75.50.Lk, 05.70.Jk, 75.40.Mg, 77.80.Bh

\section{INTRODUCTION}

Spin glasses 1 are the prototype models for disordered systems investigated extensively in statistical physics during the last three decades. These systems exhibit complex energy landscapes resulting in many interesting phenomena, like glassy behavior and aging. Despite much effort, many questions are still open.

For two-dimensional spin glasses with only nearestneighbor interactions, it is now clear that no stable spinglass phase at finite temperature exists 2.3 .4 .5 .6 . This means that they are paramagnetic at any finite temperature and that they exhibit spin-glass behavior only at $T=0$. Thus, it is widely believed, that their low-temperature behavior can well be described by the droplet theory ${ }^{7,8,9}$. The droplet picture assumes that the low-temperature behavior is governed by droplet-like excitations, where excitations of linear spatial extent $l$ typically cost an energy of order $l^{\theta}$. Thus in the thermodynamic limit the excitations which flip a finite fraction of the spins cost an infinite amount of energy if $\theta>0$. For the two-dimensional spin glass with Gaussian interactions, since it exhibits no order at $T>0, \theta<0$ holds 10 . Furthermore it is usually assumed that the energy of different types of excitations, e.g. droplets and domain walls, induced by changing the boundary conditions, are described by the same exponent $\theta$. Indeed, recently is has been confirmed by calculating exact ground states $^{11}$ that $\theta=-0.282(2)$ for droplet and domain-wall excitations ${ }^{12,13}$. For domain walls, small sizes are sufficient to see the asymptotics, hence similar values have been found previously ${ }^{2,14,15,16,17,18,19}$ in this case. On the other hand, for some droplet excitations, this behavior is only visible for not-too small system sizes $L \geq 50$, which explains why in a similar preceding study ${ }^{20}$ of smaller sizes an apparently different exponent close to $\theta=-0.47$ has been found. For other types of droplet-like excitations, the exponent $\theta \approx-0.28$ is again already visible for small sizes ${ }^{21,22}$. Hence, the behavior at zero temperature seems to be relatively clear ${ }^{23}$.

The situation is different for the small but finitetemperature behavior. In case the correlation length $\xi$ diverges algebraically for $T \rightarrow 0$ like $\xi \sim T^{-\nu}$, the critical exponent $\nu$ of the correlation length is related through a simple renormalization argument ${ }^{14.15}$ to the droplet exponent $\theta$ via $\theta=-1 / \nu$. Several numerical studies to obtain $\nu$ at finite temperatures have been performed. Using transfer-matrix calculations of long $\left(L_{x}\right.$ up to $\left.10^{6}\right)$ and narrow $\left(L_{y}\right.$ up to 11$)$ stripes, values of ${ }^{26} \nu=2.96(22)$ respectively ${ }^{27} \nu=4.2(5)$ have been found. For small $(L=10)$ square systems ${ }^{28}$, a value of $\nu=2.1(1)$ was found. Also a couple of Monte Carlo (MC) simulations have been performed. For small sizes $(L=12)$ and relative large temperatures $T \geq 1$, a value of $\nu=3.6(2)$ has been found ${ }^{2}$. Later, for similar system sizes but lower temperatures, a value of $\nu=1.8(4)$ was obtained 29 . Furthermore, a cluster Monte Carlo simulation of three large samples $(L=128)$ in the temperature range $T \in[0.4,1]$ has bee performed, resulting 30 in $\nu=2.0(2)$.

Since the finite-temperature studies performed so far suffer from either too small systems or too large temperatures (or both), we have performed a Monte-Carlo study of large systems up to size $L=75$. By applying a recently developed cluster algorithm ${ }^{5}$, in connection with an extension presented below, we are able to equilibrate the system at much smaller temperatures than it was possible before. Parallel and independently of us, H.G. Katzgraber, L.W. Lee and A.P. Young performed a related study ${ }^{31}$ using a similar algorithm. They focus on the direct calculation of the correlation length, while we study here other thermodynamic quantities like the Binder parameter, the spin-glass susceptibility, the distribution of overlaps, the overlap with the ground state and the specific heat, and we infer from these results the asymptotic behavior of the correlation length indirectly.

The model we study consists of $N=L^{2}$ Ising spins 
$S_{i}= \pm 1$ on a square lattice with the Hamiltonian

$$
\mathcal{H}=-\sum_{\langle i, j\rangle} J_{i j} S_{i} S_{j}
$$

where the sum runs over all pairs of nearest neighbors $\langle i, j\rangle$ and the $J_{i j}$ are quenched random variables which are distributed according to a Gaussian distribution with zero mean and unit variance. In the following, we denote the thermal average by $\langle\ldots\rangle$ and the quenched-disorder average by $[\ldots]_{J}$. Periodic boundary conditions in both directions are applied.

The rest of the paper is organized as follows. First we explain the algorithm we have applied and show that it is able to equilibrate the system. In the main part, we present our results for the different thermodynamic quantities mentioned above. In the last section, we summarize our results.

\section{ALGORITHM}

We have made extensive MC simulations of our system. To reach equilibrium down to very low temperatures for large system sizes, we have used a recently developed cluster algorithm (details can be found elsewhere ${ }^{5}$ ). To speed up equilibration at very low temperatures we have devised and used a new procedure. It consists in maintaining a list of the lowest-energy elementary excitations, and to use flipping them as MC moves. This procedure works as follows:

1. Compute the ground state of the system. To do this we used a heuristic renormalization-group based algorithm ${ }^{32.33}$. To test the efficiency of the method, we checked, for systems of size $L=100$ with open boundaries, that this algorithm systematically finds the true ground state by comparing with the result of an exact algorithm ${ }^{11}$ (which works only for planar graphs, i.e. not with fully periodic boundary conditions). Moreover during the production runs using the MC simulations, we never found excitations with negative energy, which confirms that the true ground state has been found.

2. During the equilibration phase, we systematically compare the low temperature $(T \leq 0.2)$ configurations with the ground state. They differ by clusters that are flipped. We maintain a list of the lowestenergy excitations thus found (we keep up to 10000 of such excitations for the largest system). Note that we consider only elementary excitations whose boundary is connected (two clusters flipped inside one another define two independent elementary excitations, not one). Then we introduce (in addition to the cluster algorithm) a new Monte Carlo move at low temperature $(T \leq 0.2)$ : choose an elementary excitation in the list and try to flip it using the Metropolis criterion. As soon as the list no

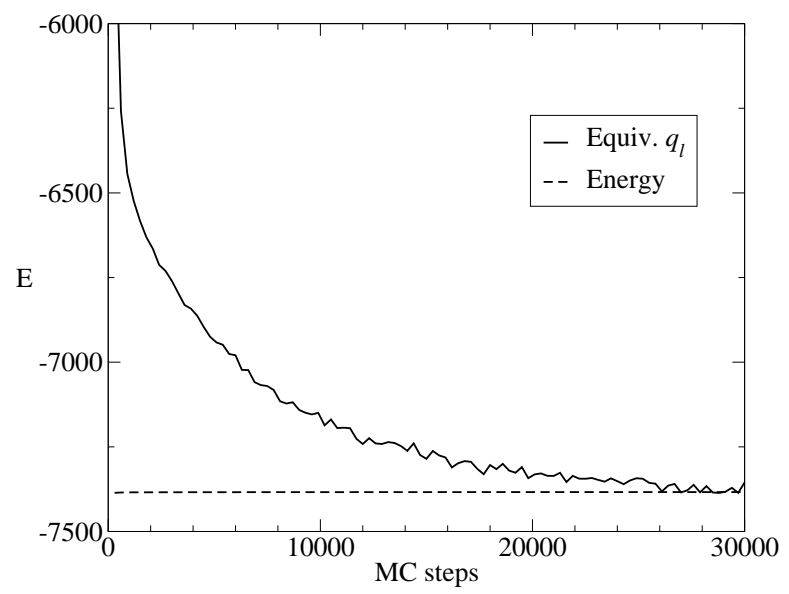

FIG. 1: Sample equilibration test for $L=75$ and $\beta=5$ averaged over 200 samples, the curves (Eq. 2) start to overlap around the equilibration time (here $25000 \mathrm{MC}$ steps).

longer evolves (i.e. when all the first excitations have been found) these moves trivially respect detailed balance.

3. During the production phase, we no longer try to find new excitations, we simply use the cluster algorithm together with single-spin flips.

Note that we obtain a list of the first elementary excitations as a by-product of this algorithm. To check that equilibrium has been reached, we have used the criterion described in Ref. 34, which is based on the following identity valid for a Gaussian distribution of the interactions:

$$
[E]_{J}=-\beta N_{l}\left(1-\left[\left\langle q_{l}\right\rangle\right]_{J}\right)
$$

where $E=\langle\mathcal{H}\rangle$ is the average energy, $\beta=1 / T$ the inverse temperature, $N_{l}=2 N$ is the number of bonds, and $q_{l}$ denotes the link overlap between two independently chosen configurations $\left\{S_{i}^{\alpha}\right\},\left\{S_{i}^{\beta}\right\}$ for the same disorder:

$$
q_{l}=\frac{1}{N_{l}} \sum_{\langle i, j\rangle} S_{i}^{\alpha} S_{j}^{\alpha} S_{i}^{\beta} S_{j}^{\beta} .
$$

In Fig. 11we show how both sides of Eq. 2] evolve during equilibration. The system can be considered equilibrated when the curves start to overlap. Note that within our algorithm, the temperature, where equilibration takes in longest time, is not the lowest temperature, because the excitation flips are most efficient at the lowest temperatures. For example at the lowest temperature, the ground state may be found after the very first step of the algorithm because all excitations present in the starting configurations can be flipped at once. 


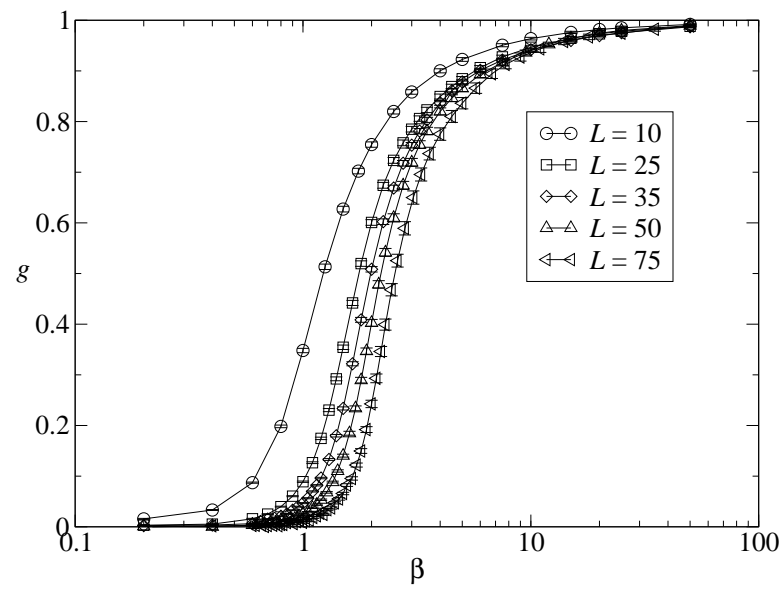

FIG. 2: Binder cumulant $g$ as a function of inverse temperature $\beta$ for different system sizes L.

\section{RESULTS}

We considered the following sizes for our simulations: $L=10,25,35,50$ and 75 over a very large range of temperatures $0.02 \leq T \leq 5$. We respectively treated 1000, 1000, 1000, 500, 200 samples for the different sizes. We simulated at different values of the temperature, the number of different values ranging from 19 to 59 (with increasing size). For each sample, we simulated 64 independent configurations at each temperature.

To study our system, we have measured different quantities and averaged over the different samples. Our first quantity of interest is the Binder cumulant ${ }^{36.37} \mathrm{~g}$ defined by

$$
g=\frac{1}{2}\left[3-\frac{\left\langle q^{4}\right\rangle}{\left\langle q^{2}\right\rangle^{2}}\right]_{J} .
$$

Here $q$ is the overlap between two independent equilibrated configurations $\left\{S_{i}^{\alpha}\right\}$ and $\left\{S_{i}^{\beta}\right\}$ of the same disorder realization

$$
q=\frac{1}{N} \sum_{i} S_{i}^{\alpha} S_{i}^{\beta}
$$

In the thermodynamic limit, the Binder cumulant is zero in the paramagnetic phase and around one in the spinglass phase. To study $g$ at finite sizes, we consider the divergence of the correlation length $\xi$ when approaching the transition temperature $T_{c}$

$$
\xi \sim\left(T-T_{c}\right)^{-\nu}
$$

Since $g$ does not show any critical behavior near the critical point, and according to the basic assumption that it is a function of the relation of system size $L$ to the correlation length $\xi, g$ scales as:

$$
g \sim \tilde{g}(L / \xi)=\tilde{g}\left(L\left(T-T_{c}\right)^{\nu}\right) .
$$

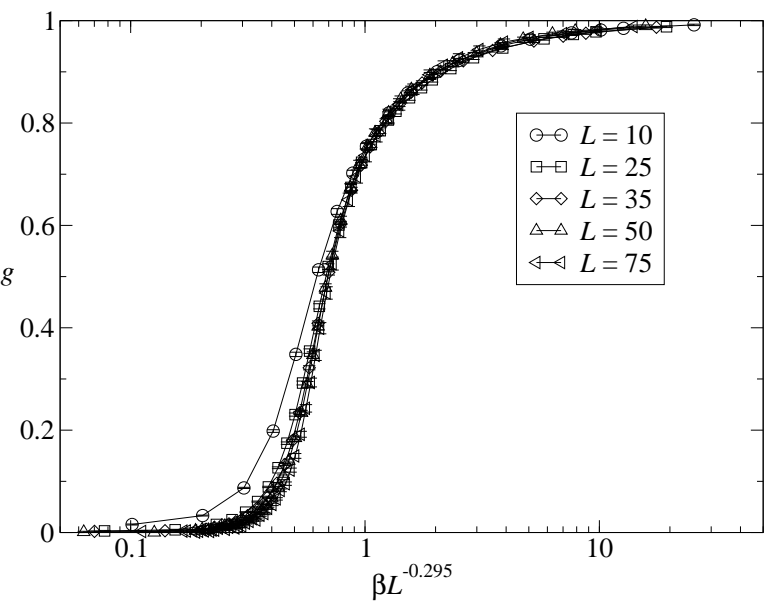

FIG. 3: Scaling of the Binder cumulant in the critical region obtained by plotting $g$ as a function of the rescaled inverse temperature $\beta L^{-1 / \nu}$ using $1 / \nu=0.295$

Hence, $g$ is independent of $L$ at $T=T_{c}$ which allows the location of $T_{c}$ : the curves for different system sizes $L$ should intersect at $T_{c}$. In Fig. 2 we show the value of $g$ as a function of $\beta$ for different system sizes $L$. The curves for $g$ converge to a crossing point at very low temperature which indicate that we most probably have $T_{c}=0$, in accordance to the recent believe $2,3,4,5,6$.

As stressed in the introduction, the value of $\nu$ has been an open question for a long time. The reason for this is the presence of large finite-size corrections to scaling. We see this, when trying to perform a finite-size scaling plot, i.e. when plotting $g$ as a function of $\beta L^{-1 / \nu}$ for a suitably chosen value of $\nu$. In fact, no value for $\nu$ allows the whole curves to collapse on a master curve. One has to select only a domain of parameters near the critical regime, namely large $L$ and small $T$ (and thus large $g$ ). Keeping only $L \geq 35$ and $g \geq 0.5$ we find that $1 / \nu \simeq$ $0.295(\nu \simeq 3.39)$. The scaling plot for this value is shown on Fig. 3 (note that we show all the data points, but only those in the range mentioned above where used to find the exponent): for large sizes and low temperatures, a very good data collapse is obtained.

To confirm this value of $\nu$ and to estimate the error, we need to somehow quantify the quality of the collapse of the curves. To do this, we use a procedure similar to one proposed by Kawashima and Ito 38 which we detail in the appendix. Using this procedure, we define a function $S(\nu)$, measuring the quality of the fit as a function of the chosen value of $\nu$. $S(\nu)$ should be around one if the collapse is good (taking the error bars into account) and much larger otherwise, it behaves somehow like a $\chi^{2}$ test. In Fig. 4 we show the value of $S(\nu)$ for the scaling according to Eq. 7. again we used only the data close to $T=0$. We see that the minimum corresponds to $S \simeq$ 1.28 which is good and we can also have an estimation for the error on $\nu: 1 / \nu \simeq 0.295 \pm 0.03$.

In Fig. 3] we see that the high temperature part, which 


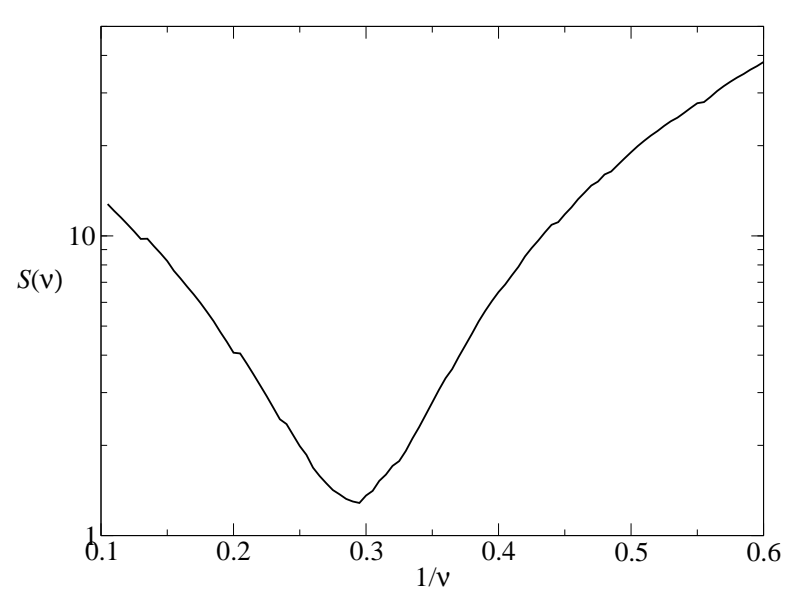

FIG. 4: Quality function $S(\nu)$ for the Binder-cumulant scaling. The minimum gives the estimation for the exponent $\nu$.

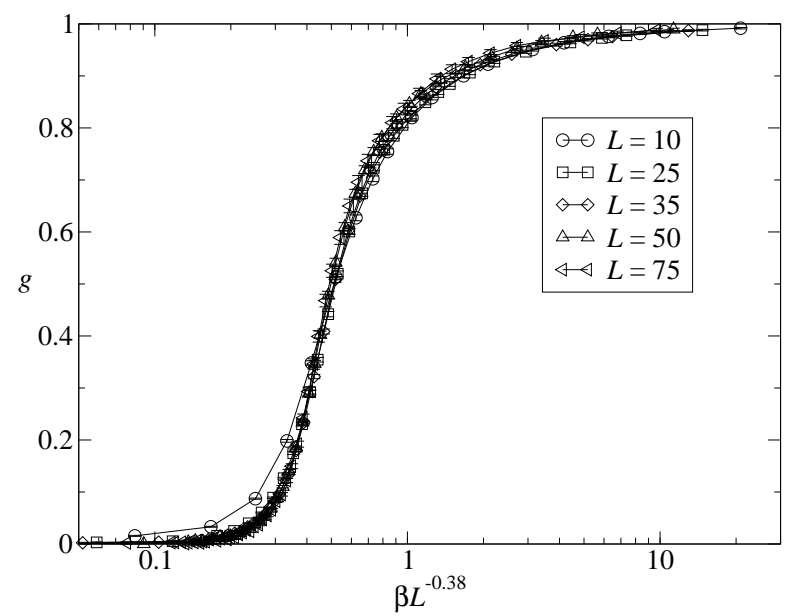

FIG. 5: Scaling of the Binder cumulant out of the critical region, when choosing $1 / \nu=0.38$.

was not used to obtain $\nu$, does not scale well. It is as if it requires another value of the exponent. In fact, if we use only data for which $L \geq 25$ and $0.1 \leq g \leq 0.5$ we find $1 / \nu \simeq 0.38$ (and $S=6.6$ which is not good) the resulting plot is shown in Fig. 5] The scaling is not good but it explains why such a high value of $1 / \nu$ appears in previous papers: large system sizes, low temperatures and a good criterion for equilibration are necessary to obtain valid results.

We now turn to the spin-glass susceptibility

$$
\chi_{\mathrm{sg}}=L^{d}\left[\left\langle q^{2}\right\rangle\right]_{J}
$$

(here $d=2$ ). Since the ground state of Ising spin glasses with a Gaussian distribution of the interactions is unique (i.e. $q=1$ ), the susceptibility shows ${ }^{28.29}$ the following finite-size behavior at low temperatures:

$$
\chi_{\mathrm{sg}} \sim L^{2} \tilde{\chi}(L / \xi) \sim L^{2} \tilde{\chi}\left(L T^{\nu}\right)
$$

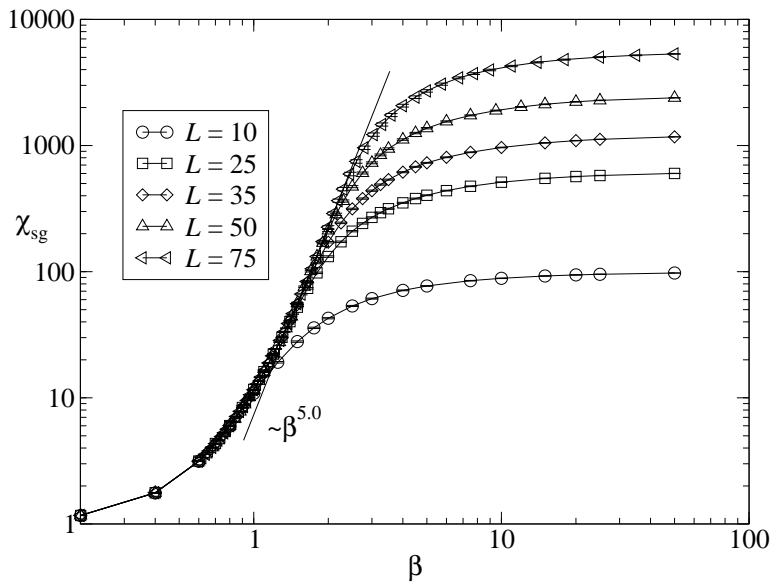

FIG. 6: The spin-glass susceptibility $\chi_{\mathrm{sg}}$ as a function of inverse temperature $\beta$ for different system sizes $L$.

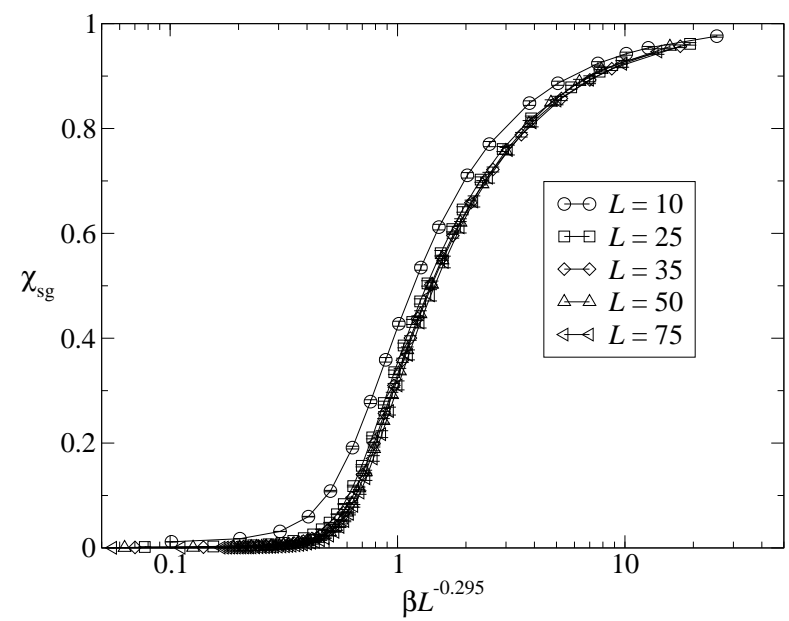

FIG. 7: Scaling of the spin-glass susceptibility $\chi_{\mathrm{sg}}$

(with $\tilde{x}(L / \xi)$ going to a constant for $T \rightarrow 0$ ). A little bit away from the critical region, where the correlation length is small compared to the system size, the susceptibility should not show any system-size dependence, hence the $L^{2}$ factor must cancel out $\left(\tilde{\chi}(x) \sim x^{-2}\right)$, and we obtain

$$
\chi_{\mathrm{sg}} \sim T^{-2 \nu}
$$

at large $L$ and finite $T$. We show our result for $\chi_{\text {sg }}$ in Fig. 6. The line added corresponds to a power law with exponent 5.0 which corresponds to $1 / \nu=0.4$. The line does not fit the data very well and there is some upward curvature of the data which indicates a larger value for the true exponent. This is fully compatible with the previous results, because $1 / \nu \simeq 0.295$ results in $2 \nu \simeq 6.8$. To test the prediction in Eq. 9] we show in Fig. [7] plot of $\chi / L^{2}$ as a function of $\beta L^{-1 / \nu}$. We get a quality $S=1.64$ of the finite-size scaling when considering data with $L \geq 35$ and $\chi_{\mathrm{sg}} / L^{2} \geq 0.3$ which means that the scaling is good. 


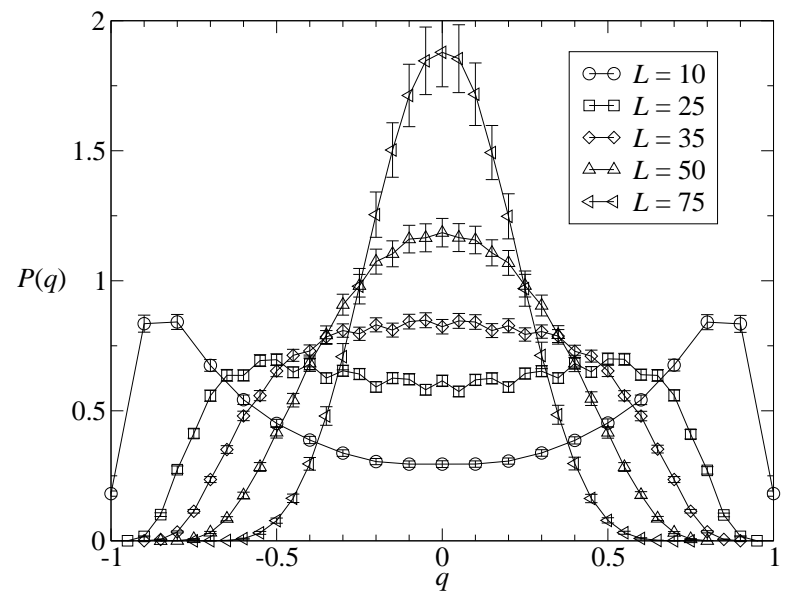

FIG. 8: The distribution $P(q)$ of overlaps for different system sizes at inverse temperature $\beta=2$.

We also studied the full distribution $P(q)$ of overlaps. The data at $\beta=2$ is shown in Fig. [8] We observe that the distributions are perfectly symmetric with respect to $q=0$, which is another indication that our simulation is well equilibrated. Furthermore, we can see a crossover from a two-peak structure for small system sizes to a trivial distribution as the size is increased. This again is fully compatible with the notion that there is no ordered phase a low temperatures except at $T=0$, hence the system is paramagnetic at finite temperatures.

We furthermore looked at the overlap of the equilibrated configurations with the ground-state

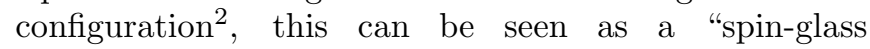
magnetization"

$$
q_{\mathrm{gs}}=\left[\left\langle\left|\frac{1}{N} \sum_{i} S_{i} S_{i}^{0}\right|\right\rangle\right]_{J},
$$

where $S_{i}^{0}$ denotes one of the two ground states. This is expected $^{2}$ to scale as

$$
q_{\mathrm{gs}} \sim \tilde{q_{\mathrm{gs}}}(L / \xi) \sim \tilde{q_{\mathrm{gs}}}\left(L T^{\nu}\right) .
$$

The raw data is shown in Fig. 9 while the data rescaled according to Eq. 12 with $1 / \nu=0.295$ is shown in Fig.10 When considering data with $L \geq 35$ and $q_{\mathrm{gs}} \geq 0.5$ we find quality $S=2.50$. The quality of this scaling is lower than the one for the Binder cumulant, nevertheless, the result still supports the findings from above. Interestingly, in Ref. 2 a similar value $1 / \nu=0.28$ was already found whereas only small systems $L \leq 12$ at high temperatures $(\beta<1.4)$ where studied.

We also studied the specific heat

$$
c=\frac{1}{N}\left[\frac{d E}{d T}\right]_{J}=\frac{\beta^{2}}{N}\left[\left\langle(\mathcal{H}-\langle\mathcal{H}\rangle)^{2}\right\rangle\right]_{J} .
$$

Although the specific heat is not expected to show any singularity for the spin-glass transition, it is nevertheless

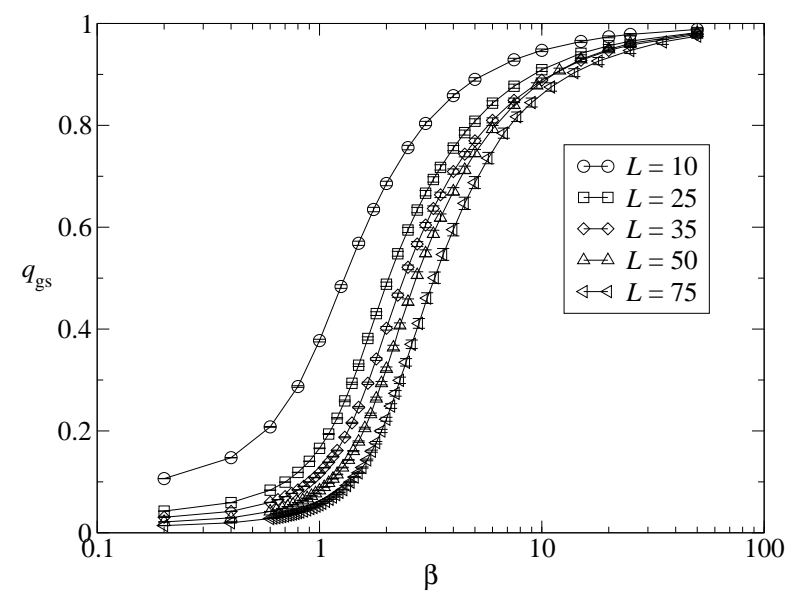

FIG. 9: The overlap $q_{\mathrm{gs}}$ with the ground state as a function of the inverse temperature for different system sizes $L$.

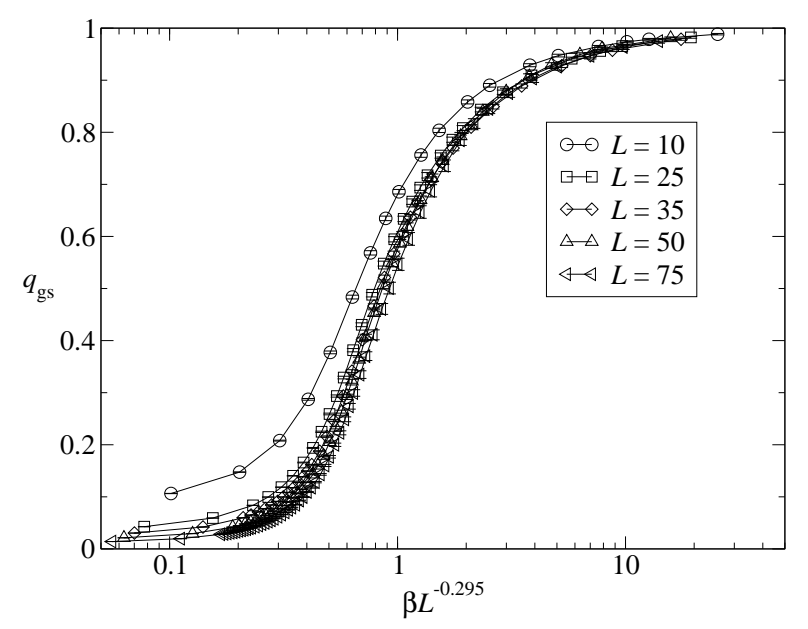

FIG. 10: Scaling plot of the overlap with the ground state: $q$ as a function of $\beta L^{-1 / \nu}$, with $1 / \nu=0.295$.

interesting to compare with previous studies. The data is shown in Fig. 11] The specific heat presents a finite peak around $\beta=0.82$ known as the "Schottky anomaly". The decay of the specific heat at small $T$ goes roughly as $c \sim T$ similarly to previous studies using transfermatrix calculations on long stripes ${ }^{26}$ respectively hierarchical lattices ${ }^{35}$. Nevertheless the data is not perfectly described by a power low even at such low temperatures and it is not clear what the asymptotic behavior truely is.

Finally, we have also directly evaluated the correlation length $\xi$ by measuring spin-spin correlations as a function of the separation of the spins and fitting suitable functions to the data. Since the behavior of the correlation is already studied in detail in Ref. 31, we do not go into details here. We only mention that our results are fully compatible with the results of Ref. 31: when going to large system sizes and studying low temperatures, we 


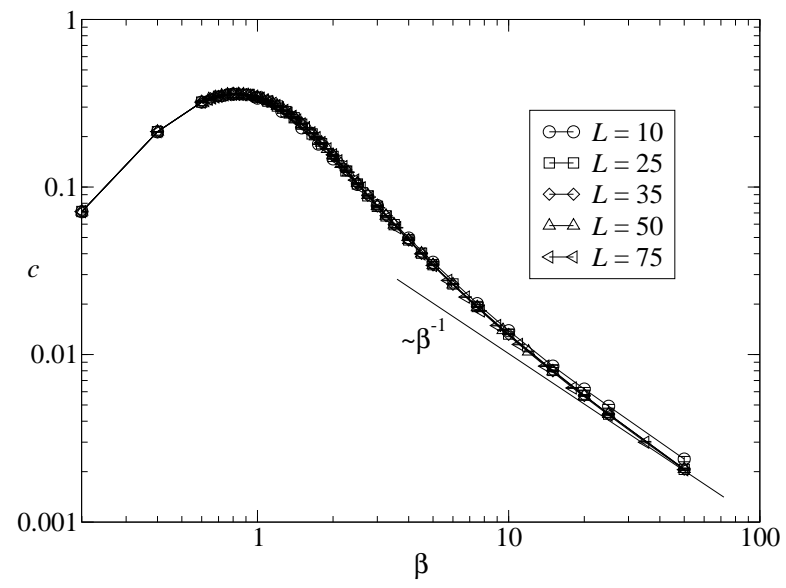

FIG. 11: The specific heat $c$ as a function of inverse temperature $\beta$ for different system sizes $L$. The straight line is a putative asymptotic behavior $c \sim T$ when $T \rightarrow 0$.

observe a behavior compatible with $-1 / \nu=-0.295$.

\section{SUMMARY AND CONCLUSIONS}

We have studied the low-temperature behavior of twodimensional spin glasses with a Gaussian distribution of the interactions. Using a sophisticated cluster algorithm in combination with exploring lowest-energy excitations close to $T=0$, we were able to equilibrate large system sizes up to $L=75$ down to very small temperatures $T=0.02$.

We have studied several thermodynamical quantities, like Binder cumulant, susceptibility, distribution of overlaps, overlap with the ground state and specific heat. Our main findings are as follows. From the Binder cumulant, and the distribution of overlaps, we see that no stable spin-glass phase at finite temperature exists, i.e. $T_{c}=0$ in accordance to recent studies. From the scaling behavior of the Binder cumulant and the susceptibility, we find that the correlation length diverges algebraically for $T \rightarrow 0$, in contrast to the model with a bimodal distribution of the interactions ${ }^{5}$. The main open question was, whether the exponent $\theta \approx-0.29$, describing the scaling of droplet and domain-wall excitations, is equal to $-1 / \nu$, $\nu$ being the exponent of the correlation length. Our results $-1 / \nu=-0.295(30)$ obtained for large sizes and at low temperatures indeed support $\theta=-1 / \nu$.

To conclude, when taking ground-state studies into account: the thermodynamic behavior of the twodimensional Gaussian Ising spin glass is trivial for finite but low temperatures and governed by one single exponent $-1 / \nu=\theta \approx-0.29$, as predicted by the droplet picture simple renormalization arguments.

\section{ACKNOWLEDGMENTS}

The authors thank H.G. Katzgraber, L.W. Lee, and A.P. Young for helpful discussions and sending us their preprint prior to submission. AKH obtained financial support from the VolkswagenStiftung (Germany) within the program "Nachwuchsgruppen an Universitäten". This work was supported in part by the European Community's Human Potential Program under contract number HPRN-CT-2002-00307, DYGLAGEMEM.

\section{APPENDIX A: DETERMINING THE QUALITY OF THE SCALING LAWS}

To determine the quality of the scaling laws we need some quality criterium that somehow measures the distance of the data to the master curve. The difficulty arise from the fact that the master curve is unknown and must be determined from the data. Some years ago, Kawashima and Ito ${ }^{38}$ proposed a method. We present here a refinement which, according to our experience, seemed to be stabler and more precise.

After applying the scaling law, we have $k$ sets of points. Each set is composed of $n_{i}$ points $(i=1 \ldots k)$ of the form $\left(x_{i j}, y_{i j}, d y_{i j}\right)$ with $d y$ being the standard error on $y$ and $j=1 \ldots n_{i}$. In the following we suppose that $x_{i 1}<x_{i 2}<\ldots<x_{i n_{i}}$. We define the quality as

$$
S=\frac{1}{\mathcal{N}} \sum_{i, j} \frac{\left(y_{i j}-Y_{i j}\right)^{2}}{d y_{i j}^{2}+d Y_{i j}^{2}},
$$

where $Y_{i j}$ and $d Y_{i j}$ are the estimated position and standard error of the master curve at $x_{i j} . \mathcal{N}$ is the number of terms in the sum (we only consider the terms for which $Y_{i j}$ and $d Y_{i j}$ are defined).

To define $Y_{i j}$ and $d Y_{i j}$, we first select a set of points as follow: in each set $i^{\prime} \neq i$, we select two points $j^{\prime}$ and $j^{\prime}+1$ such that $x_{i^{\prime} j^{\prime}} \leq x_{i j} \leq x_{i^{\prime}\left(j^{\prime}+1\right)}$, if there are no such points in a set, we do not select any point from that set (the set does not determine the position of the master curve for this value of $x$ ). If this procedure selects no point at all then $Y_{i j}$ and $d Y_{i j}$ are undefined for point $i j$ and it does not contribute to $S$ (this happens if set $i$ is alone in this region of $x$ and is the master curve by itself). We now compute the linear fit through the selected points $\left(x_{l}, y_{l}, d y_{l}\right), l=1 \ldots m$ and $Y_{i j}$ is the value of that straight line at $x_{i j}$ and $d Y_{i j}$ is the associated standard error, namely

$$
Y_{i j}=\frac{K_{x x} K_{y}-K_{x} K_{x y}}{\Delta}+x_{i j} \frac{K K_{x y}-K_{x} K_{y}}{\Delta}
$$

and

$$
d Y_{i j}^{2}=\frac{1}{\Delta}\left(K_{x x}-2 x_{i j} K_{x}+x_{i j}^{2} K\right)
$$

with $w_{l}=1 / d y_{l}^{2}, K=\sum w_{l}, K_{x}=\sum w_{l} x_{l}, K_{y}=$ $\sum w_{l} y_{l}, K_{x x}=\sum w_{l} x_{l}^{2}, K_{x y}=\sum w_{l} x_{l} y_{l}$ and $\Delta=$ $K K_{x x}-K_{x}^{2}$. 
The quality $S$ measures the mean square distance to the master curve of the sets in unit of standard errors. It should thus be around one if the data really collapse to a single curve and much larger otherwise.
1 Reviews on spin glasses can be found in: K. Binder and A.P. Young, Rev. Mod. Phys. 58, 801 (1986); M. Mézard, G. Parisi, M.A. Virasoro, Spin glass theory and beyond, (World Scientific, Singapore 1987); K.H. Fisher and J.A. Hertz, Spin Glasses, (Cambridge University Press, Cambridge 1991); A.P. Young (ed.), Spin glasses and random fields, (World Scientific, Singapore 1998); N. Kawashima and H. Rieger, preprint cond-mat/0312432

2 H. Rieger, L. Santen, U. Blasum, M. Diehl, M. Jünger, and G. Rinaldi, J. Phys. A 29, 3939 (1996).

${ }^{3}$ N. Kawashima and H. Rieger, Europhys. Lett. 39, 85 (1997).

4 A.K. Hartmann and A.P. Young, Phys. Rev B 64, 180404 (2001).

5 J. Houdayer, Eur. Phys. Jour. B 22, 479 (2001).

6 A.C. Carter, A.J. Bray, and M.A. Moore, Phys. Rev. Lett. 88, 077201 (2002).

7 W.L. McMillan, J. Phys. C 17, 3179 (1984).

8 A.J. Bray and M.A. Moore, in Glassy Dynamics and Optimization, edited by J.L. van Hemmen and I. Morgenstern, (Springer, Berlin, 1986).

9 D.S. Fisher and D.A. Huse, Phys. Rev. Lett. 56, 1601 (1986); Phys. Rev. B 38, 386 (1988).

10 For a bimodal distribution of the bonds, $\theta=0$, see Ref. 4 , corresponding to an exponential divergence of the correlation length ${ }^{5}$.

11 A.K. Hartmann and H. Rieger, Optimization Algorithms in Physics, (Wiley-VCH, Berlin 2001).

12 A.K. Hartmann, A.J. Bray, A.C. Carter, M.A. Moore, and A.P. Young, Phys. Rev. B 66, 224401 (2002).

13 A.K. Hartmann and M.A. Moore, Phys. Rev. Lett. 90, 127201 (2003).

14 A.J. Bray and M. Moore, J. Phys. C 17, L463 (1984).

15 W.L. McMillan, Phys. Rev. B 29, 4026 (1984).

16 W.L. McMillan, Phys. Rev. B 30, 476 (1984).

17 M. Cieplak and J.R. Banavar, J. Phys. A 23, 4385 (1990).

18 F. Matsubara, T. Shirakura, and M. Shiomi, Phys. Rev. B 58, R11821 (1998).
19 N. Kawashima and T. Aoki, J. Phys. Soc. Jpn., 69, Suppl. A, 169 (2000).

20 N. Kawashima, J. Phys. Soc. Jpn. 69, 987 (2000).

21 A.K. Hartmann and A.P. Young, Phys. Rev. B 66, 094419 (2002).

22 L. Berthier and A. P. Young, J. Phys. A 36, 10835 (2003).

${ }^{23}$ Although recently, when studying ${ }^{24}$ first excitations of small systems $(L \leq 12)$, again an exponent close to -0.47 has been found. This seems also to depend on the boundary conditions ${ }^{25}$.

24 M. Picco, F. Ritort and M. Sales, Phys. Rev. B 67, 184421 (2003).

25 A.K. Hartmann and M. Moore, to appear in Phys. Rev. B, preprint cond-mat/0310080

${ }^{26}$ H.-F. Cheung and W.L. McMillan, J. Phys. C 16, 7033 (1983)

27 D.A. Huse and I. Morgenstern, Phys. Rev. B 32, 3032 (1985).

28 N. Kawashima, N. Hatano, and M. Suzuki, J. Phys. A 25, 4985 (1992).

29 M. Ney-Nifle and A.P. Young, J. Phys. A 30, 5311 (1997).

30 S. Liang, Phys. Rev. Lett. 69, 2145 (1992).

31 H.G. Katzgraber, L.W. Lee and A.P. Young, to be published (2004).

32 J. Houdayer and O.C. Martin, Phys. Rev. Lett. 83, 1030 (1999).

33 J. Houdayer and O.C. Martin, Phys. Rev. E 64, 056704 (2001).

34 H.G. Katzgraber, M. Palassini, and A.P. Young, Phys. Rev. B 63, 184422 (2001).

35 R.F.S. Andrade, E. Nogueira Jr., and S. Coutinho, Phys. Rev. B 68, 104523 (2003).

36 K. Binder, Z. Phys. B 43, 119 (1981).

37 R.N. Bhatt and A.P. Young, Phys. Rev. Lett. 54, 924 (1985); Phys. Rev. B 37, 5606 (1988).

38 N. Kawashima and N. Ito: J. Phys. Soc. Jpn. 62, 435 (1993). 